\title{
Integral terminal sliding mode control for nonlinear systems
}

\author{
GUO Jianguo*, LIU Yuchao, and ZHOU Jun \\ Institute of Precision Guidance and Control, Northwestern Polytechnical University, Xi' an 710072, China
}

\begin{abstract}
This paper proposes a fast integral terminal sliding mode (ITSM) control method for a cascaded nonlinear dynamical system with mismatched uncertainties. Firstly, an integral terminal sliding mode surface is presented, which not only avoids the singularity in the traditional terminal sliding mode, but also addresses the mismatched problems in the nonlinear control system. Secondly, a new ITSM controller with finite convergence time based on the backstepping technique is derived for a cascaded nonlinear dynamical system with mismatched uncertainties. Thirdly, the convergence time of ITSM is analyzed, whose convergence speed is faster than those of two nonsingular terminal sliding modes. Finally, simulation results are presented in order to evaluate the effectiveness of ITSM control strategies for mismatched uncertainties.
\end{abstract}

Keywords: terminal sliding mode (TSM), finite-time convergence, mismatched disturbance, Lyapunov stability, nonlinear systems.

DOI: $10.21629 /$ JSEE.2018.03.14

\section{Introduction}

Mismatched disturbances widely exist in various control engineering systems, such as the kinematical and dynamical equations of the near space vehicles [1], the integrated guidance and control model of the near space interceptor in the three-dimensional space [2], and the mathematics model of the hypersonic reentry vehicle [3]. These nonlinear control systems with mismatched disturbances could be easily developed as a kind of cascaded nonlinear form with mismatched uncertainties.

In terms of dealing with the mismatched disturbance, sliding mode control (SMC) and some related credible techniques have been applied cooperatively, which means it combines different methods with SMC to address the mismatched uncertainties.

It is well-known that SMC is a robust theory with regard to matched uncertainties. However, there are some drawbacks with the classical SMC that guarantees the

\footnotetext{
Manuscript received September 06, 2016.

*Corresponding author.

This work was supported by the National Natural Science Foundation of China (61473226).
}

finite converge time to the origin on sliding mode and deals with mismatched systems. To address this finite converge time problem, the terminal sliding mode control (TSMC) is presented by Venkataraman and Gulati [4], which applies a nonlinear sliding hyperplane instead of a linear sliding surface and thus improves the convergence performance. After that, many scholars issue their conclusions and gain research results based on this thesis, such as Song, et al. [5], Aghababa, \& Mohammad [6], Khari, et al. [7], Zhong, et al. [8]. However, it is still unsolved that the singularity in TSMC caused by the derivative of fractional power functions that work as sliding hyperplanes. The new nonsingular terminal sliding modes (TSMs) are proposed to avoid the above problem according to some direct methods instead of the initial TSM [9,10]. Singularity is dealt with indirect methods by switching from the initial TSM to general sliding manifold which can be linear or nonlinear $[11-14]$. The available nonsingular TSMC methods are merely for second-order systems [15].

Castaños and Fridman put forward a combination of $H_{\infty}$ and integral SMC to reduce the influences of the mismatched uncertainties [16]. Besides, to solve the mismatched systems, the backstepping method is used with SMC. Dynamic adaptive backstepping and first and second order SMC are used for the collaboration of triangular and nontriangular uncertain observable minimum phase nonlinear systems [17]. Another approach regarding the problem of mismatched uncertainty compensation is based on the nonlinear block controllable form [18]. A sliding mode controller is designed with the combination of block control, a sigmoid approximation to the integral SMC and nested SMC [19]. Although these methods are asymptotically tracking, this can be eliminated by TSMC.

This paper presents a fast integral terminal sliding mode (ITSM) control method in respect to a cascaded nonlinear uncertain mismatched dynamical system. It is a new nonsingular TSMC method with a convergence speed faster than that of a normal fast TSM at any point by introducing an ITSM. Generally, the method not only guarantees states convergence in the sliding mode within finite time, 
but also accelerates the convergence rate to reach the equilibrium point.

This paper is structured as follows: Section 1 is regarding to the theory introduction. Section 2 shows problem formulations and assumptions. In Section 3 methodologies are divided into two parts, which mainly focuses on the development and characteristics of finite time as well as the controller based on backstepping. Particularly, singularity is solved with a 2-sliding algorithm. In Section 4, the time taken to reach the manifold from any initial system states and the time taken to reach the equilibrium point in the sliding mode are guaranteed to be finite. Then, the proposed ITSM controller is applied to two examples with simulation in Matlab, where simulation results are used to validate the analysis.

\section{Problem formulations and assumptions}

A cascaded uncertain mismatched nonlinear system is defined as follows [1]:

$$
\left\{\begin{array}{l}
\dot{\boldsymbol{x}}_{i}=\boldsymbol{f}_{i}\left(\boldsymbol{x}_{i}\right)+\boldsymbol{g}_{i}\left(\boldsymbol{x}_{i}\right) \boldsymbol{x}_{i+1}+\boldsymbol{\Delta}_{i}(t), \quad 1 \leqslant i \leqslant \bar{l}-1 \\
\dot{\boldsymbol{x}}_{\bar{l}}=\boldsymbol{f}_{\bar{l}}\left(\boldsymbol{x}_{\bar{l}}\right)+\boldsymbol{g}_{\bar{l}}(\boldsymbol{x}) \boldsymbol{u}+\boldsymbol{\Delta}_{\bar{l}}(t) \\
\boldsymbol{y}=\boldsymbol{x}_{1}
\end{array}\right.
$$

where $\boldsymbol{x}=\left[\boldsymbol{x}_{1}, \boldsymbol{x}_{2}, \ldots, \boldsymbol{x}_{\bar{l}}\right]$ is the system state vector and $\boldsymbol{x}_{i} \in \mathbf{R}^{n_{i}}, \boldsymbol{x} \in \mathbf{R}^{n}, n_{1}+n_{2}+\cdots+n_{\bar{l}}=n$, $\boldsymbol{u} \in \mathbf{R}^{m}$ is the system input, $\boldsymbol{y}$ is the system output, $\boldsymbol{f}_{j}\left(\boldsymbol{x}_{j}\right) \in \mathbf{R}^{n_{j}}(1 \leqslant j \leqslant \bar{l})$ are smooth functions, $\boldsymbol{g}_{i}\left(\boldsymbol{x}_{i}\right) \in \mathbf{R}^{n_{i} \times n_{i+1}}(1 \leqslant i \leqslant \bar{l}-1)$ and $\boldsymbol{g}_{\bar{l}}(\boldsymbol{x}) \in \mathbf{R}^{n_{\bar{l}} \times m}$, $\boldsymbol{\Delta}_{j}(t)(1 \leqslant j \leqslant \bar{l})$ is the mismatched uncertainties consisting of external load disturbance and parameter uncertainties.

The following assumptions and lemmas are given for the plant (1).

Assumption $1 \boldsymbol{g}_{i}\left(\boldsymbol{x}_{i}\right)(1 \leqslant i \leqslant \bar{l}-1)$ and $\boldsymbol{g}_{\bar{l}}(\boldsymbol{x})$ are nonsingular.

Assumption $2\left\|\boldsymbol{\Delta}_{j}(t)\right\| \leqslant d_{j}, 1 \leqslant j \leqslant \bar{l}, \forall t \geqslant 0$, $d_{j}>0$ is a known constant.

Lemma $1^{[10]}$ The equilibrium point $q=0$ is globally finite-time stable for any given initial condition $q(0)=$ $q_{0}$, if a Lyapunov description can be obtained as $\dot{V}(q)+$ $\alpha V(q)+\beta V^{\eta}(q) \leqslant 0, \alpha>0, \beta>0,0<$ $\eta<1$, and the settling time can be given by $T \leqslant$ $\frac{1}{\alpha(1-\eta)} \ln \left(\frac{\alpha V^{\eta-1}\left(q_{0}\right)+\beta}{\beta}\right)$, where $V\left(q_{0}\right)$ is the initial value of $V(q)$.

\section{Methodologies}

\subsection{ITSM}

An ITSM surface for $\boldsymbol{x}_{1}$ in (1) is defined as follows:

$$
\boldsymbol{s}=\boldsymbol{x}_{1}+\int_{0}^{t}\left[\boldsymbol{K}_{1} \boldsymbol{x}_{1}^{l}+\boldsymbol{K}_{2} \boldsymbol{x}_{1}+\varepsilon \operatorname{sgn}\left(\boldsymbol{x}_{1}\right)\right] \mathrm{d} t
$$

where $x_{1}^{l}=\left[x_{1}^{l}, x_{2}^{l}, \ldots, x_{n_{1}}^{l}\right]^{\mathrm{T}}, l=\frac{p}{q}>1$ and $p, q$ are positive odd integers, $\boldsymbol{K}_{1}, \boldsymbol{K}_{2}$ and $\varepsilon$ are positive diagonal matrices.

Theorem 1 The equilibrium point $\boldsymbol{x}_{1}=\mathbf{0}$ is finite-time stable for the ITSM (2) if $s=0$, i.e., the system output $y$ will converge to zero in finite time.

Proof With regard to the proposed sliding mode surface in (2), When system state $\boldsymbol{x}_{1}$ moves on the sliding mode surface $s=0$ in (2), $x_{1}$ can be written as follows:

$$
\dot{\boldsymbol{x}}_{1}=-\boldsymbol{K}_{1} \boldsymbol{x}_{1}^{l}-\boldsymbol{K}_{2} \boldsymbol{x}_{1}-\varepsilon \operatorname{sgn}\left(\boldsymbol{x}_{1}\right) .
$$

A Lyapunov function candidate is selected as

$$
\bar{V}\left(\boldsymbol{x}_{1}\right)=\boldsymbol{x}_{1}^{\mathrm{T}} \boldsymbol{x}_{1} / 2 .
$$

As a consequence, the time derivative of $\bar{V}\left(\boldsymbol{x}_{1}\right)$ can be obtained as follows:

$$
\dot{\bar{V}}\left(\boldsymbol{x}_{1}\right)=\boldsymbol{x}_{1}^{\mathrm{T}} \dot{\boldsymbol{x}}_{1} \leqslant-k_{1} \sum_{i=1}^{n_{1}} x_{i}^{l+1}-k_{2}\left\|\boldsymbol{x}_{1}\right\|^{2}-\varepsilon\left\|\boldsymbol{x}_{1}\right\|
$$

where $k_{1}, k_{2}$ and $\varepsilon$ are the smallest eigenvalues of $\boldsymbol{K}_{1}, \boldsymbol{K}_{2}$ and $\varepsilon$, respectively.

Considering that $p, q$ are positive odd integers, the sum $p+q$ is even integer, and $x_{i}^{l+1} \geqslant 0$ due to $l+1=\frac{p+q}{q}$.

Thus (5) can be rewritten as follows:

$$
\dot{\bar{V}}\left(\boldsymbol{x}_{1}\right) \leqslant-2 k_{2} \bar{V}\left(\boldsymbol{x}_{1}\right)-\sqrt{2} \varepsilon \bar{V}^{1 / 2}\left(\boldsymbol{x}_{1}\right) .
$$

Obviously, (6) satisfies

$$
\dot{\bar{V}}\left(\boldsymbol{x}_{1}\right)+\alpha \bar{V}\left(\boldsymbol{x}_{1}\right)+\beta \bar{V}^{\eta}\left(\boldsymbol{x}_{1}\right) \leqslant 0
$$

where $\alpha=2 k_{2}>0, \beta=\sqrt{2} \varepsilon>0$ and $0<\eta=1 / 2<1$. According to Lemma $1, \bar{V}\left(\boldsymbol{x}_{1}\right)$ converges to zero within the time $t$, where $t=\frac{1}{\alpha(1-\eta)} \ln \left(\frac{\alpha \bar{V}^{\eta-1}\left(\boldsymbol{x}_{10}\right)+\beta}{\beta}\right)$.

As a result, the system state $\boldsymbol{x}_{1}$ will converge to zero in finite time when system state $\boldsymbol{x}_{1}$ moves on the sliding mode surface $s=0$, that is to say, the system output $\boldsymbol{y}$ will converge to zero in finite time.

Remark 1 When the system state $\boldsymbol{x}_{1}$ is replaced by the siliding surface $s$, a new reaching law can be obtained according to the sliding surface (2).

$$
\dot{s}=-K_{1} s^{l}-K_{2} s-\varepsilon \operatorname{sgn}(s)
$$

\subsection{Backstepping ITSM control}

Based on the proposed ITSM (2), the ITSM control for mismatched nonlinear system (1) is shown in the following. 
The aim is to design the new ITSM controller which could eliminate the effect of mismatched uncertainties, and the system output $\boldsymbol{y}$ could track the desired output $\boldsymbol{y}_{m}$ in finite time under the ITSM controller.

The ITSM for system (1) in the case $\bar{l} \geqslant 1$ is designed as follows:

$\boldsymbol{s}_{j}=\boldsymbol{e}_{j}+\int_{0}^{t}\left[\boldsymbol{K}_{j 1} \boldsymbol{e}_{j}^{l}+\boldsymbol{K}_{j 2} \boldsymbol{e}_{j}+\boldsymbol{\varepsilon}_{j} \operatorname{sgn}\left(\boldsymbol{e}_{j}\right)\right] \mathrm{d} t, \quad 1 \leqslant j \leqslant \bar{l}$

where $\boldsymbol{e}_{j}=\boldsymbol{x}_{j}-\boldsymbol{x}_{j m}$ is the state error, $\boldsymbol{x}_{1 m}=\boldsymbol{y}_{m}$, $\boldsymbol{x}_{(i+1) m}=\boldsymbol{x}_{(i+1) c}(1 \leqslant i \leqslant \bar{l}-1), \boldsymbol{x}_{(i+1) c}$ is the designed virtual control, $\boldsymbol{e}_{j}^{l}=\left[e_{1}^{l}, e_{2}^{l}, \ldots, e_{n_{j}}^{l}\right]^{\mathrm{T}}, \boldsymbol{K}_{j 1}, \boldsymbol{K}_{j 2}$ and $\varepsilon_{j}$ are positive diagonal matrices.

Theorem 2 The equilibrium point $e_{1}=\mathbf{0}$ is globally finite-time stable for the uncertain mismatched nonlinear system (1) with the proposed ITSM surface (8), if the cascaded virtual control $\boldsymbol{x}_{(i+1) c}(1 \leqslant i \leqslant \bar{l}-1)$ is defined as

$$
\begin{gathered}
\boldsymbol{x}_{(i+1) c}= \\
-\boldsymbol{g}_{i}^{-1}\left(\boldsymbol{x}_{i}\right)\left[\boldsymbol{f}_{i}\left(\boldsymbol{x}_{j}\right)+\boldsymbol{K}_{i 1} \boldsymbol{e}_{j}^{l}+\boldsymbol{K}_{i 2} \boldsymbol{e}_{j}+\boldsymbol{\varepsilon}_{i} \operatorname{sgn}\left(\boldsymbol{e}_{i}\right)-\right. \\
\left.\dot{\boldsymbol{x}}_{i c}+\boldsymbol{H}_{i 1} \boldsymbol{s}_{j}^{l}+\boldsymbol{H}_{i 2} \boldsymbol{s}+\boldsymbol{\delta}_{i} \operatorname{sgn}\left(\boldsymbol{s}_{i}\right)\right]
\end{gathered}
$$

and the control law is chosen as

$$
\begin{gathered}
\boldsymbol{u}=-\boldsymbol{g}_{\bar{l}}^{-1}(\boldsymbol{x})\left[\boldsymbol{f}_{\bar{l}}(\boldsymbol{x})+\boldsymbol{K}_{\bar{l} 1} \boldsymbol{e}_{\bar{l}}^{l}+\boldsymbol{K}_{\bar{l} 2} \boldsymbol{e}_{\bar{l}}+\boldsymbol{\varepsilon}_{\bar{l}} \operatorname{sgn}\left(\boldsymbol{e}_{\bar{l}}\right)-\right. \\
\left.\dot{\boldsymbol{x}}_{\bar{l} \mathrm{c}}+\boldsymbol{H}_{\bar{l} 1} \boldsymbol{s}_{n}^{l}+\boldsymbol{H}_{\bar{l} 2} \boldsymbol{s}_{n}+\boldsymbol{\delta}_{\bar{l}} \operatorname{sgn}\left(\boldsymbol{s}_{\bar{l}}\right)\right]
\end{gathered}
$$

where $\boldsymbol{x}_{1 c}=\boldsymbol{y}_{m}, \boldsymbol{x}_{n c}$ is design virtual control, $\boldsymbol{H}_{j 1}, \boldsymbol{H}_{j 2}$ and $\boldsymbol{\delta}_{j}(1 \leqslant j \leqslant \bar{l})$ are positive diagonal matrices. $\delta_{j}$ is defined as the smallest eigenvalue of $\boldsymbol{\delta}_{j}$ and $\delta_{j}>d_{j}$. Accordingly, the expected result $\boldsymbol{y}_{m}$ in finite time is tracked by the system output $\boldsymbol{y}$.

Proof The proof consists of the ITSM control and the backstepping technique.

Step 1 Let $i=1$, (1) and (9) can be written as

$$
\begin{gathered}
\dot{\boldsymbol{x}}_{1}=\boldsymbol{f}_{1}\left(\boldsymbol{x}_{1}\right)+\boldsymbol{g}_{1}\left(\boldsymbol{x}_{1}\right) \boldsymbol{x}_{2}+\boldsymbol{\Delta}_{1}(t) \\
\boldsymbol{s}_{1}=\boldsymbol{e}_{1}+\int_{0}^{t}\left[\boldsymbol{K}_{11} \boldsymbol{e}_{1}^{l}+\boldsymbol{K}_{12} \boldsymbol{e}_{1}+\boldsymbol{\varepsilon}_{1} \operatorname{sgn}\left(\boldsymbol{e}_{1}\right)\right] \mathrm{d} t
\end{gathered}
$$

where $\boldsymbol{e}_{1}=\boldsymbol{y}-\boldsymbol{y}_{m}=\boldsymbol{x}_{1}-\boldsymbol{y}_{m}, \boldsymbol{e}_{1}^{l}=\left[e_{1}^{l}, e_{2}^{l}, \ldots, e_{n_{1}}^{l}\right]^{\mathrm{T}}$, $\boldsymbol{K}_{11}, \boldsymbol{K}_{12}$ and $\varepsilon_{1}$ are positive diagonal matrices.

Taking the derivation of $s_{1}$ results in

$$
\dot{\boldsymbol{s}}_{1}=\dot{\boldsymbol{x}}_{1}-\dot{\boldsymbol{y}}_{m}+\boldsymbol{K}_{11} \boldsymbol{e}_{1}^{l}+\boldsymbol{K}_{12} \boldsymbol{e}_{1}+\boldsymbol{\varepsilon}_{1} \operatorname{sgn}\left(\boldsymbol{e}_{1}\right) .
$$

The virtual control $\boldsymbol{x}_{2 c}$ can be defined as follows:

$$
\begin{gathered}
\boldsymbol{x}_{2 c}=-\boldsymbol{g}_{1}^{-1}\left(\boldsymbol{x}_{1}\right)\left[\boldsymbol{f}_{1}\left(\boldsymbol{x}_{1}\right)+\boldsymbol{K}_{11} \boldsymbol{e}_{1}^{l}+\boldsymbol{K}_{12} \boldsymbol{e}_{1}+\varepsilon_{1} \operatorname{sgn}\left(\boldsymbol{e}_{1}\right)-\right. \\
\left.\dot{\boldsymbol{y}}_{m}+\boldsymbol{H}_{11} \boldsymbol{s}_{1}^{l}+\boldsymbol{H}_{12} \boldsymbol{s}+\boldsymbol{\delta}_{1} \operatorname{sgn}\left(\boldsymbol{s}_{1}\right)\right]
\end{gathered}
$$

where $\boldsymbol{H}_{11}, \boldsymbol{H}_{12}$ and $\boldsymbol{\delta}_{1}$ are positive diagonal matrices.
Considering that the corresponding Lyapunov function candidate is $V_{1}=s_{1}^{\mathrm{T}} s_{1} / 2$, time derivate of $V_{1}$ along (15) is

$$
\dot{V}_{1}=\boldsymbol{s}_{1}^{\mathrm{T}}\left[-\boldsymbol{H}_{11} \boldsymbol{s}_{1}^{l}-\boldsymbol{H}_{12} \boldsymbol{s}-\boldsymbol{\delta}_{1} \operatorname{sgn}\left(\boldsymbol{s}_{1}\right)+\boldsymbol{\Delta}_{1}(t)\right] .
$$

According to Assumption 2, (14) can be rewritten as follows:

$$
\begin{gathered}
\dot{V}_{1} \leqslant-h_{11} \sum_{i=1}^{n_{i}} s_{1}^{l+1}-h_{12}\left\|s_{1}\right\|^{2}-\left(\delta_{1}-d_{1}\right)\left\|s_{1}\right\| \leqslant \\
-2 h_{12} V_{1}-\sqrt{2}\left(\delta_{1}-d_{1}\right) V_{1}^{1 / 2}
\end{gathered}
$$

where $h_{11}, h_{12}$ and $\delta_{1}$ are the smallest eigenvalues of $\boldsymbol{H}_{11}$, $\boldsymbol{H}_{12}$ and $\boldsymbol{\delta}_{1}$, respectively, and $\delta_{1}>d_{1}$.

The parameters are satisfied as $\alpha=2 h_{12}>0, \beta=$ $\sqrt{2}\left(\delta_{1}-d_{1}\right)>0$ and $\eta=1 / 2$ respectively due to (17) and Lemma $1, V_{1}$ converges to zero in finite time, i.e, $\boldsymbol{s}_{1}$ converges to zero in finite time.

When $s_{1}=0$, tracking error $\boldsymbol{e}_{1}$ moves on the sliding mode surface $s_{1}=0$ according to Theorem 1, it is clear that the tracking error $\boldsymbol{e}_{1}$ goes to zero in finite time, i.e., the system output $\boldsymbol{y}$ can track the desired output $\boldsymbol{y}_{m}$ within finite time.

Step $k$ A similar procedure is employed recursively for each step $k(2 \leqslant k \leqslant \bar{l}-1)$.

The state equation is defined as

$$
\dot{\boldsymbol{x}}_{k}=\boldsymbol{f}_{k}\left(\boldsymbol{x}_{k}\right)+\boldsymbol{g}_{k}\left(\boldsymbol{x}_{k}\right) \boldsymbol{x}_{k+1}+\boldsymbol{\Delta}_{k}(t) .
$$

The ITSM for (18) is selected as

$$
\boldsymbol{s}_{k}=\boldsymbol{e}_{k}+\int_{0}^{t}\left[\boldsymbol{K}_{k 1} \boldsymbol{e}_{k}^{l}+\boldsymbol{K}_{k 2} \boldsymbol{e}_{k}+\boldsymbol{\varepsilon}_{k} \operatorname{sgn}\left(\boldsymbol{e}_{k}\right)\right] \mathrm{d} t
$$

where $\boldsymbol{e}_{k}=\boldsymbol{x}_{k}-\boldsymbol{x}_{k c}$ is the state error, $\boldsymbol{x}_{k c}$ is the virtual control in step $k-1, \boldsymbol{e}_{k}^{l}=\left[e_{1}^{l}, e_{2}^{l}, \ldots, e_{n_{k}}^{l}\right]^{\mathrm{T}}, \boldsymbol{K}_{k 1}, \boldsymbol{K}_{k 2}$ and $\varepsilon_{k}$ are positive diagonal matrices.

There exists a Lyapunov function candidate $V_{k}=$ $\boldsymbol{s}_{k}^{\mathrm{T}} \boldsymbol{s}_{k} / 2$ based on the similar process in step 1 , and the virtual control $\boldsymbol{x}_{(k+1) c}$ is defined as follows:

$$
\begin{gathered}
\boldsymbol{x}_{(k+1) c}=-\boldsymbol{g}_{k}^{-1}\left(\boldsymbol{x}_{k}\right)\left[\boldsymbol{f}_{k}\left(\boldsymbol{x}_{k}\right)+\boldsymbol{K}_{k 1} \boldsymbol{e}_{k}^{l}+\boldsymbol{K}_{k 2} \boldsymbol{e}_{k}+\right. \\
\left.\varepsilon_{k} \operatorname{sgn}\left(\boldsymbol{e}_{k}\right)-\dot{\boldsymbol{x}}_{k c}+\boldsymbol{H}_{k 1} \boldsymbol{s}_{k}^{l}+\boldsymbol{H}_{k 2} \boldsymbol{s}_{k}+\boldsymbol{\delta}_{k} \operatorname{sgn}\left(\boldsymbol{s}_{k}\right)\right]
\end{gathered}
$$

where $\boldsymbol{H}_{k 1}, \boldsymbol{H}_{k 2}$ and $\boldsymbol{\delta}_{k}$ are positive diagonal matrices, $h_{k 2}$ and $\delta_{k}$ are the smallest eigenvalue of $\boldsymbol{H}_{k 2}$ and $\boldsymbol{\delta}_{k}$, respectively, such that $\dot{V}_{k} \leqslant-2 h_{k 2} V_{k}-\sqrt{2}\left(\delta_{k}-d_{k}\right) V_{k}^{1 / 2}$ and $\delta_{k}>d_{k}$, and system state $\boldsymbol{x}_{k}$ can track the virtual control $\boldsymbol{x}_{k c}$ in finite time due to Lemma 1.

Step $\overline{\boldsymbol{l}}$ For $\dot{\boldsymbol{x}}_{\bar{l}}=\boldsymbol{f}_{\bar{l}}(\boldsymbol{X})+\boldsymbol{g}_{\bar{l}}(\boldsymbol{X}) \boldsymbol{u}+\boldsymbol{\Delta}_{\bar{l}}(t)$, the sliding mode $s_{n}$ is chosen as (9), where $\boldsymbol{e}_{\bar{l}}=\boldsymbol{x}_{\bar{l}}-\boldsymbol{x}_{\bar{l} c}$.

The control law is satisfied as (11). Taking the derivation of $s_{n}$ yields

$$
\dot{\boldsymbol{s}}_{\bar{l}}=\boldsymbol{\Delta}_{\bar{l}}(t)-\boldsymbol{H}_{\bar{l} 1} \boldsymbol{s}_{n}^{l}-\boldsymbol{H}_{\bar{l} 2} \boldsymbol{s}_{n}-\boldsymbol{\delta}_{\bar{l}} \operatorname{sgn}\left(\boldsymbol{s}_{\bar{l}}\right) .
$$


When a Lyapunov function candidate is defined as $V_{\bar{l}}=$ $s_{\bar{l}}^{\mathrm{T}} s_{\bar{l}} / 2$ based on the similar process in step 1 , it could obtain the similar result as

$$
\dot{V}_{\bar{l}} \leqslant-2 h_{\bar{l} 2} V_{\bar{l}}-\sqrt{2}\left(\delta_{\bar{l}}-d_{\bar{l}}\right) V_{\bar{l}}^{1 / 2} .
$$

where $h_{\bar{l} 2}$ and $\delta_{\bar{l}}$ are the smallest eigenvalue of $\boldsymbol{H}_{\bar{l} 2}$ and $\boldsymbol{\delta}_{\bar{l}}$, respectively, and $\delta_{\bar{l}}>d_{\bar{l}}$. It can be seen that $\boldsymbol{s}_{n}$ converges to zero in finite time by Lemma 1 . Moreover, the above procedure can guarantee the system stability in finite time.

A Lyapunov function candidate is set as

$$
V=\sum_{i=1}^{\bar{l}} \boldsymbol{s}_{i}^{\mathrm{T}} \boldsymbol{s}_{i} / 2
$$

Taking the time derivative of $V$ along the system dynamics (1) leads to

$$
\begin{gathered}
\dot{V} \leqslant-h_{12}\left\|s_{1}\right\|^{2}-h_{22}\left\|s_{2}\right\|^{2}-\cdots-h_{i 2}\left\|s_{i}\right\|^{2}-\cdots- \\
h_{\bar{l} 2}\left\|s_{\bar{l}}\right\|^{2}-\bar{\delta}_{1}\left\|s_{1}\right\|-\bar{\delta}_{2}\left\|s_{2}\right\|-\cdots-\bar{\delta}_{i}\left\|s_{i}\right\|-\cdots-\bar{\delta}_{\bar{l}}\left\|s_{\bar{l}}\right\| \leqslant \\
-2 \bar{h} V-\sqrt{2} \bar{\delta} V^{1 / 2}
\end{gathered}
$$

where $\bar{h}=\min \left(h_{12}, h_{22}, \ldots, h_{\bar{l} 2}\right), \quad \bar{\delta}=$ $\min \left(\bar{\delta}_{1}, \bar{\delta}_{2}, \ldots, \bar{\delta}_{\bar{l}}\right), \bar{\delta}_{j}=\delta_{j}-d_{j}(1 \leqslant j \leqslant \bar{l})$. According to Lemma 1, the system states can converge to zero in finite time, and the system output $\boldsymbol{y}$ can track the desired output $\boldsymbol{y}_{m}$ in finite time.

Note that in the above results, it is clear that there a exists singular problem when taking the time derivative of the virtual control $\boldsymbol{x}_{k c}(2 \leqslant k \leqslant \bar{l})$ due to discontinuous signum function. In order to keep $\boldsymbol{\xi}_{k}-\boldsymbol{x}_{k c}=0$ and $\dot{\boldsymbol{\xi}}_{k}=\dot{\boldsymbol{x}}_{k c}$ in finite time [20], the auxiliary equation can be obtained based on a modified 2-sliding algorithm:

$$
\left\{\begin{array}{l}
\dot{\boldsymbol{\xi}}_{k}=\boldsymbol{x}_{k r}-\lambda_{1}\left\|\boldsymbol{z}_{k}\right\|^{1 / 2} \operatorname{sgn}\left(\boldsymbol{z}_{k}\right) \\
\dot{\boldsymbol{x}}_{k r}=-\lambda_{2} \operatorname{sgn}\left(\boldsymbol{z}_{k}\right)
\end{array}\right.
$$

where $\boldsymbol{z}_{k}=\boldsymbol{\xi}_{k}-\boldsymbol{x}_{k c}, \lambda_{i}>0(i=1,2)$.

Using $\dot{\boldsymbol{\xi}}_{n}$ and $\dot{\boldsymbol{x}}_{n c}$ in the control law (11), the singular problem can be solved.

Remark 2 As the function of $\operatorname{sgn}(\cdot)$ in (11) is uncontinous, the continous function of $\operatorname{sat}(\cdot)$ is used in the control law (11) to avoid the chattering. As a consequence, the control law (11) can be rewritten as follows:

$$
\begin{gathered}
\boldsymbol{u}=-\boldsymbol{g}_{\bar{l}}^{-1}(\boldsymbol{x})\left[\boldsymbol{f}_{\bar{l}}(\boldsymbol{x})+\boldsymbol{K}_{\bar{l} 1} \boldsymbol{e}_{\bar{l}}^{l}+\boldsymbol{K}_{\bar{l} 2} \boldsymbol{e}_{\bar{l}}+\varepsilon_{\bar{l}} \operatorname{sat}\left(\boldsymbol{e}_{\bar{l}}\right)-\right. \\
\left.\dot{\boldsymbol{x}}_{\bar{l} c}+\boldsymbol{H}_{\bar{l} 1} \boldsymbol{s}_{n}^{l}+\boldsymbol{H}_{\bar{l} 2} \boldsymbol{s}_{n}+\boldsymbol{\delta}_{\bar{l}} \operatorname{sat}\left(\boldsymbol{s}_{\bar{l}}\right)\right]
\end{gathered}
$$

\section{Finite-time convergence analysis}

The ITSM control method for multi-input multi-output (MIMO) system has been presented in the above section, and convergence in finite time of ITSM control is analyzed in this section. In order to explain explicitly the issue, the analyse of convergence for the ITSM (2) focuses on that the dimension of $s(t)$ equals to one, i.e.,

$$
s=x+\int_{0}^{t}\left[\bar{k}_{1} x^{l}+\bar{k}_{2} x+\bar{\varepsilon}_{1} \operatorname{sgn}(x)\right] \mathrm{d} t
$$

where $x \in \mathbf{R}, s \in \mathbf{R}, \bar{k}_{1}, \bar{k}_{2}$ and $\bar{\varepsilon}_{1}$ are positive constants, and the reaching law is defined as

$$
\dot{s}=-\bar{k}_{3} s^{l}-\bar{k}_{4} s-\bar{\varepsilon}_{2} \operatorname{sgn}(s)
$$

where $\bar{k}_{3}, \bar{k}_{4}$ and $\bar{\varepsilon}_{2}$ are positive constants.

The convergence time consists of the time in the reaching phase and the time in the sliding phase. It is difficult to obtain the time in the reaching phase due to (28). However, it is easy to get the convergence time between the interval $s(0) \neq 0$ and $s=0$, where $l=3$.

Without loss of generity, assuming $s(t)>0$, and $s(0)>$ 1 , and if $\bar{\varepsilon}_{2} \operatorname{sgn}(s)$ is unaccounted, it is obtained as

$$
\dot{s}+\bar{k}_{3} s^{l}+\bar{k}_{4} s=0 .
$$

When $l=3$, (29) is a Bernoulli differential equation and the corresponding solution is

$$
s(t)=\left[\mathrm{e}^{-2 \bar{k}_{4} t}\left(1 / s^{2}(0)+\bar{k}_{3} / \bar{k}_{4}\right)-\bar{k}_{3} / \bar{k}_{4}\right]^{-1 / 2} .
$$

The time from $s(0)>1$ to $s(t)=1$ is obtained as $t_{1}=\ln \left[\left(\bar{k}_{4} / s^{2}(0)+\bar{k}_{3}\right) /\left(\bar{k}_{4}+\bar{k}_{3}\right)\right] / 2 \bar{k}_{4}$ from (30).

When $s(0) \leqslant 1$, without considering $\bar{k}_{4} s$, the reaching law (28) can be written as

$$
\dot{s}+\bar{k}_{3} s^{l}+\bar{\varepsilon}_{2}=0 .
$$

When $l=3$ with $\bar{\varepsilon}_{2}=\bar{k}_{3} \lambda^{3}$, where $\lambda$ is a positive number, (31) is an Abel differential equation and it can be obtained as

$$
\begin{gathered}
\ln \left(\frac{s(0)+\lambda}{s(t)+\lambda}\right)+\frac{1}{2} \ln \left(s^{2}(t)-\lambda s(t)+\lambda^{2}\right)+ \\
\frac{5}{\sqrt{3}}\left[\arctan \left(\frac{\lambda-2 s(0)}{\sqrt{3} \lambda}\right)-\arctan \left(\frac{\lambda-2 s(t)}{\sqrt{3} \lambda}\right)\right]= \\
3 \bar{k}_{3} \lambda^{2} t .
\end{gathered}
$$

The time from $s(0) \leqslant 1$ to $s(t)=0$ is obtained as $t_{2}=$ $\frac{1}{3 \bar{k}_{3} \lambda^{2}}\left\{\ln (\lambda+s(0))+\frac{5}{\sqrt{3}}\left[\arctan \left(\frac{\lambda-2 s(0)}{\sqrt{3} \lambda}\right)-\frac{\pi}{6}\right]\right\}$ from (32).

The sliding mode $s$ under the reaching law (28) with $l=2$ at any point $s(0)$ will reach zero in finite time $t_{r}$ which needs to satisfy

$$
t_{r}<\left\{\begin{array}{l}
t_{1}+t_{2}, \quad|s(0)|>1 \\
t_{2},|s(0)| \leqslant 1
\end{array}\right.
$$


where $t_{1}$ and $t_{2}$ satisfy

$$
\begin{gathered}
t_{1}=\ln \left[\left(\bar{k}_{4} / s^{2}(0)+\bar{k}_{3}\right) /\left(\bar{k}_{4}+\bar{k}_{3}\right)\right] / 2 \bar{k}_{4} \\
t_{2}=\frac{1}{3 \bar{k}_{3} \lambda^{2}}\{\ln (\lambda+s(0))+ \\
\left.\frac{5}{\sqrt{3}}\left[\arctan \left(\frac{\lambda-2 s(0)}{\sqrt{3} \lambda}\right)-\frac{\pi}{6}\right]\right\} .
\end{gathered}
$$

Similarly, the time in the sliding phase can be obtained due to (27). When $s=0$, it is obtained as

$$
\dot{x}=-\bar{k}_{1} x^{l}-\bar{k}_{2} x-\bar{\varepsilon}_{1} \operatorname{sgn}(x) .
$$

The state $x$ on the sliding mode with $l=3$ and $\bar{\varepsilon}_{1}=$ $\bar{k}_{1} \lambda^{3}$ at any point $x(0)$ will reach zero in finite time $t_{s}$ which satisfies

$$
t_{s}<\left\{\begin{array}{l}
t_{3}+t_{4}, \quad|x(0)|>1 \\
t_{4}, \quad|x(0)| \leqslant 1
\end{array}\right.
$$

where $t_{3}$ and $t_{4}$ satisfy

$$
\begin{gathered}
t_{3}=\ln \left[\left(\bar{k}_{2} / x^{2}(0)+\bar{k}_{1}\right) /\left(\bar{k}_{2}+\bar{k}_{1}\right)\right] / 2 \bar{k}_{2} \\
t_{4}=\frac{1}{3 \bar{k}_{1} \lambda^{2}}\{\ln (\lambda+x(0))+ \\
\left.\frac{5}{\sqrt{3}}\left[\arctan \left(\frac{\lambda-2 x(0)}{\sqrt{3} \lambda}\right)-\frac{\pi}{6}\right]\right\} .
\end{gathered}
$$

It is easily obtained that the system state $x$ can converges to zero within finite time $t_{r}+t_{s}$ when the sliding mode surface is chosen as (27) and the reaching law is used as (28).

Remark 3 The convergence time of the system state on the sliding mode (27) from $x\left(t_{0}\right) \neq 0$ to zero is shorter than that of other TSMs.

For example, the fast TSM can be described by using the following nonlinear equation [21]:

$$
s=\dot{x}+\alpha x+\beta x^{q / p}
$$

where $\alpha>0, \beta>0$, and the positive odd integers $p$ and $q$ are selected such that $1<p / q<2$.

When the system state on the sliding mode and $\left|x\left(t_{0}\right)\right|>1$, the convergence rate of ITSM (27) and TSM (41) satisfies

$$
\bar{k}_{1}|x(t)|^{l}+\bar{k}_{2}|x(t)|>\beta|x(t)|^{q / p}+\alpha|x(t)|
$$

when $\bar{k}_{1}=\beta$ and $\bar{k}_{2}=\alpha$. When $\left|x\left(t_{0}\right)\right| \leqslant 1$, the convergence rate of ITSM (27) and TSM (41) satisfies

$$
\bar{k}_{2}|x(t)|+\bar{\varepsilon}_{1}>\beta|x(t)|^{q / p}+\alpha|x(t)|
$$

when $\bar{k}_{2}=\alpha$ and $\bar{\varepsilon}_{1}=\beta$.
Obviously, the convergence time of the system state on the $\operatorname{ITSM}(27)$ from $x\left(t_{0}\right) \neq 0$ to zero is shorter than that of TSM (34) due to the fact that the convergence rate of $\operatorname{ITSM}(27)$ is more than that of TSM (41).

The same result can be obtained for TSM [22], which is defined by

$$
s=x+\int_{0}^{t}\left(\beta x^{l}+\gamma x^{\bar{l}}\right) \mathrm{d} t
$$

where $\gamma>0$ and $0<\bar{l}<1$.

When the state on the sliding mode, when $\left|x\left(t_{0}\right)\right|>1$, the convergence rate of ITSM (27) and TSM (44) satisfies

$$
\bar{k}_{1}|x(t)|^{l}+\bar{k}_{2}|x(t)|>\beta|x(t)|^{l}+\gamma|x(t)|^{\bar{l}}
$$

when $\bar{k}_{1}=\beta$ and $\bar{k}_{2}=\gamma$. When $\left|x\left(t_{0}\right)\right| \leqslant 1$, the convergence rate of ITSM (27) and TSM (44) satisfies

$$
\bar{k}_{2}|x(t)|^{l}+\bar{\varepsilon}_{1}>\beta|x(t)|^{l}+\gamma|x(t)|^{\bar{l}}
$$

when $\bar{k}_{2}=\beta$ and $\bar{\varepsilon}_{1}=\gamma$.

\section{Simulation}

Two examples are presented in order to test the validity of above ITSM control.

Firstly, in order to analyze the effectiveness of proposed ITSM control and compare ITSM with other TSMs, the simple three-order dynamical system is considered as follows.

Example 1 Three-order system

$$
\left\{\begin{array}{l}
\dot{x}_{1}=x_{2}+0.1 \cos x_{1}+0.05 \sin (2 t) \\
\dot{x}_{2}=x_{3}+0.05 \sin (10 t) \\
\dot{x}_{3}=u+0.1 \sin (20 t)
\end{array} .\right.
$$

Since the sigularity occurs in the TSM control, the modified fast TSM (MFTSM) [13] can be described as follows:

$$
s_{\mathrm{MFTSM} 1}=x_{2}+x_{1}+\beta_{2}\left(x_{1}\right)
$$

where

$$
\begin{aligned}
& \beta_{2}\left(x_{1}\right)=\left\{\begin{array}{l}
x_{1}^{\frac{3}{5}}, \quad \bar{s}_{2}=0 \text { or } \bar{s}_{2} \neq 0,\left|x_{1}\right| \geqslant 1 \\
\frac{7}{5} x_{1}-\frac{2}{5} \operatorname{sgn}\left(x_{1}\right) x_{1}^{2}, \quad \bar{s}_{2} \neq 0,\left|x_{1}\right| \leqslant 1,
\end{array}\right. \\
& \bar{s}_{2}=x_{2}+x_{1}+x_{1}^{\frac{3}{5}} .
\end{aligned}
$$

TSM and ITSM are defined respectively as follows:

$$
\begin{gathered}
s_{\mathrm{TSM} 1}=x_{1}+\int_{0}^{t}\left[x_{1}^{\frac{3}{5}}+x_{1}^{\frac{5}{3}}\right] \mathrm{d} t \\
s_{\text {ITSM } 1}=x_{1}+\int_{0}^{t}\left[x_{1}^{\frac{5}{3}}+x_{1}+\operatorname{sgn}\left(x_{1}\right)\right] \mathrm{d} t .
\end{gathered}
$$

The three different controllers can be described by the backstepping method as follows. 
MFTSM controller is defined as

$$
u=\dot{x}_{M 3 c}-e_{M 2}-0.2 s a t\left(e_{M 2}\right)
$$

where

$$
\begin{gathered}
e_{M 2}=x_{3}-x_{M 3 c} \\
x_{M 3 c}=-x_{2}-\frac{3}{5} x_{2} x_{1}^{-\frac{2}{5}}-s_{\text {MFTSM } 1}-0.2 \operatorname{sat}\left(s_{\text {MFTSM } 1}\right) .
\end{gathered}
$$

When $\bar{s}_{2}=0$ or $\bar{s}_{2} \neq 0$ and $\left|x_{1}\right| \geqslant 1$,

$$
\begin{gathered}
x_{M 3 c}=-x_{2}-\frac{7}{5} x_{2}+\frac{2}{5} x_{1} x_{2} \operatorname{sat}\left(x_{1}\right)- \\
s_{\text {MFTSM } 1}-0.2 \operatorname{sat}\left(s_{\text {MFTSM } 1}\right)
\end{gathered}
$$

when $\bar{s}_{2} \neq 0$ and $\left|x_{1}\right| \leqslant 1$.

TSM controller is defined as

$$
u=\dot{x}_{T 3 c}-e_{T 2}^{\frac{3}{5}}-e_{T 2}^{\frac{5}{3}}-s_{\mathrm{TSM} 3}-0.2 \operatorname{sat}\left(s_{\mathrm{TSM} 3}\right)
$$

where

$$
\begin{gathered}
x_{T 3 c}=\dot{x}_{T 2 c}-e_{T 1}^{\frac{3}{5}}-e_{T 1}^{\frac{5}{3}}-s_{\mathrm{TSM} 2}-0.2 \operatorname{sat}\left(s_{\mathrm{TSM} 2}\right) \\
x_{T 2 c}=-x_{1}^{\frac{3}{5}}-x_{1}^{\frac{5}{3}}-s_{\mathrm{TSM} 1}-0.2 \operatorname{sat}\left(s_{\mathrm{TSM} 1}\right) \\
s_{\mathrm{TSM} 3}=e_{T 2}+\int_{0}^{t}\left[e_{T 2}^{\frac{3}{5}}+e_{T 2}^{\frac{5}{3}}\right] \mathrm{d} t, \quad e_{T 2}=x_{3}-x_{T 3 c} \\
s_{\mathrm{TSM} 2}=e_{T 1}+\int_{0}^{t}\left[e_{T 1}^{\frac{3}{5}}+e_{T 1}^{\frac{5}{3}}\right] \mathrm{d} t, \quad e_{T 1}=x_{2}-x_{T 2 c} .
\end{gathered}
$$

ITSM controller is defined as

$$
u=\dot{x}_{I 3 c}-e_{I 2}^{\frac{5}{3}}-e_{I 2}-\operatorname{sat}\left(e_{I 2}\right)-s_{\text {ITSM3 }}-0.2 \operatorname{sat}\left(s_{\text {ITSM } 3}\right)
$$

where

$$
\begin{gathered}
x_{I 3 c}=\dot{x}_{I 2 c}-e_{I 1}^{\frac{5}{3}}-e_{I 1}- \\
\operatorname{sat}\left(e_{I 1}\right)-s_{\text {ITSM } 2}-0.2 \operatorname{sat}\left(s_{\text {ITSM } 2}\right) \\
x_{I 2 c}=-x_{1}^{\frac{5}{3}}-x_{1}-\operatorname{sat}\left(x_{1}\right)-s_{\text {ITSM } 1}-0.2 s a t\left(s_{\text {ITSM } 1}\right) \\
s_{\text {ITSM } 3}=e_{I 2}+\int_{0}^{t}\left[e_{I 2}^{\frac{5}{3}}+e_{I 2}+\operatorname{sgn}\left(e_{I 2}\right)\right] \mathrm{d} t \\
e_{I 2}=x_{3}-x_{I 3 c} \\
s_{\text {ITSM2 }}=e_{I 1}+\int_{0}^{t}\left[e_{I 1}^{\frac{5}{3}}+e_{I 1}+\operatorname{sgn}\left(e_{I 1}\right)\right] \mathrm{d} t \\
e_{I 1}=x_{2}-x_{I 2 c} .
\end{gathered}
$$

By comparing three types of controllers, it is concluded that the system state $x_{1}$ can reach the point zero under ITSM controller in finite time, as shown in Fig. 1, while the system state $x_{1}$ could not converge to zero under MFTSM controller and TSM controller due to the nonlinear function and the mismatched uncertainties.

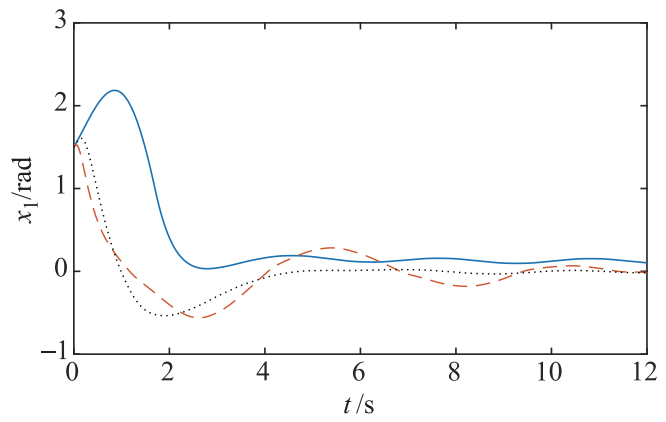

— MFTSM; $\quad---:$ TSM; $\quad$............. ITSM.

Fig. 1 States $x_{1}(t)$ under three controllers

It is obvious from Fig. 2 that the state $x_{2}$ under TSM controller does not converge the constant because the TSM controller is sensitive to the mismatched uncertainties with the sinusoidal function. In Fig. 3 the responses of the states $x_{3}$ are similar under three TSM controllers.

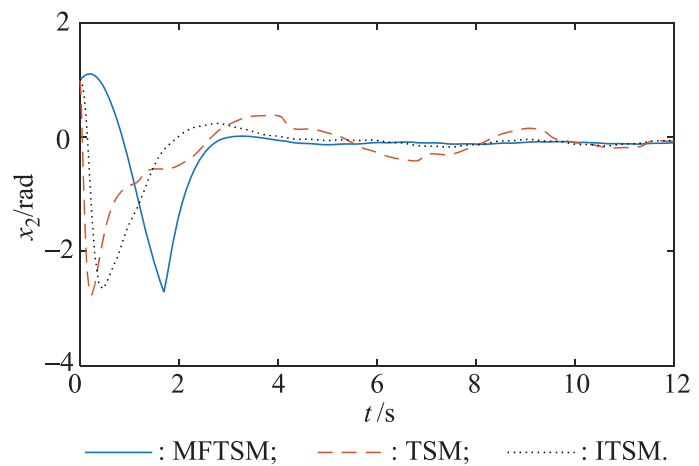

Fig. 2 States $x_{2}(t)$ under three controllers

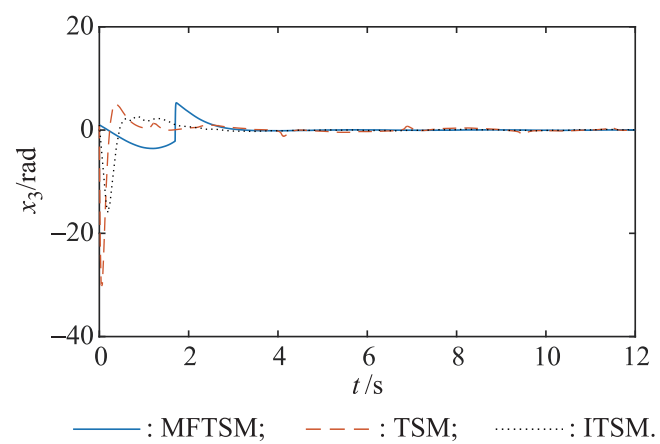

Fig. 3 States $x_{3}(t)$ under three controllers

The responses of the three control signals are provided in Fig. 4. The performances of three TSM controllers are continuous, and no singularity occurs in the case of TSM controls. Obviously, the control energy of the MFTSM controller is the minimum and that of the ITSM controller is the maximum in the intial phase.

The proposed ITSM control method could not only guarantee the state converges to zero in finite time, but also eliminate the effect of the mismatched uncertainties. 


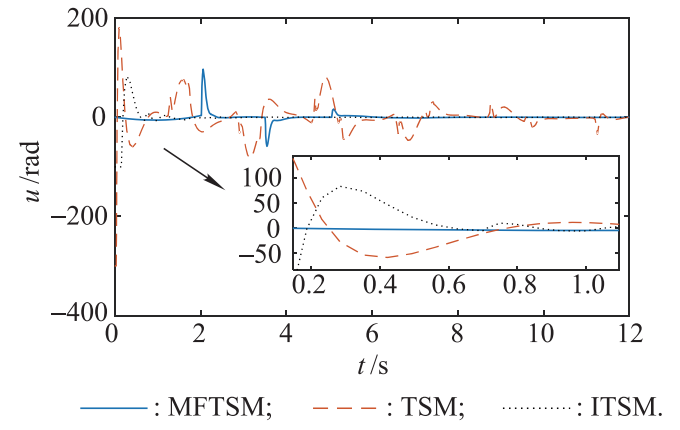

Fig. 4 The three controllers

Secondly, in order to analyze the effectiveness of the proposed ITSM control applying the control engineering system, the mathematics model of the hypersonic vehicle [3] is considered as follows.

Example 2 Consider the model of hypersonic vehicle as follows:

$$
\left\{\begin{array}{l}
\dot{x}_{1}=\boldsymbol{A}_{11} \boldsymbol{x}_{1}+\boldsymbol{A}_{12} \boldsymbol{x}_{2}+\boldsymbol{f}_{1} \\
\dot{\boldsymbol{x}}_{2}=\boldsymbol{A}_{21} \boldsymbol{x}_{1}+\boldsymbol{A}_{22} \boldsymbol{x}_{2}+\boldsymbol{B} \boldsymbol{u}+\boldsymbol{f}_{2}
\end{array}\right.
$$

where $\boldsymbol{x}_{1}=\left[\begin{array}{l}\alpha \\ \beta \\ \gamma\end{array}\right], \boldsymbol{x}_{2}=\left[\begin{array}{l}\omega_{z} \\ \omega_{y} \\ \omega_{x}\end{array}\right], \boldsymbol{u}=\left[\begin{array}{l}\delta_{z} \\ \delta_{y} \\ \delta_{x}\end{array}\right]$,

$$
\begin{array}{ccc}
\boldsymbol{A}_{11}= & {\left[\begin{array}{ccc}
b_{4} & 0 & 0 \\
0 & a_{5} & 0 \\
0 & 0 & 0
\end{array}\right],} & \boldsymbol{A}_{12}=\left[\begin{array}{ccc}
b_{3} & 0 & 0 \\
0 & a_{2} & a_{1} \\
0 & 0 & 1
\end{array}\right], \\
\boldsymbol{A}_{21}=\left[\begin{array}{ccc}
a_{4} & 0 & 0 \\
0 & b_{5} & 0 \\
c_{4} & c_{5} & 0
\end{array}\right], & \boldsymbol{A}_{22}=\left[\begin{array}{ccc}
a_{3} & 0 & 0 \\
0 & b_{2} & b_{1} \\
0 & c_{2} & c_{1}
\end{array}\right], \\
\boldsymbol{B} & =\left[\begin{array}{ccc}
a_{8} & 0 & 0 \\
0 & b_{7} & b_{6} \\
0 & c_{7} & c_{6}
\end{array}\right],
\end{array}
$$

$a_{11}=0.5, a_{12}=5, a_{13}=2 ; b_{11}=1, b_{12}=2, b_{13}=$ $3 ; a_{21}=0.5, a_{22}=5, a_{23}=2 ; b_{21}=2, b_{22}=1, b_{23}=1$; $\alpha$ is the angle of attack, $\beta$ is the sideslip angle, $\gamma$ is the roll angle, $\omega_{z}$ is the pitch angular velocity, $\omega_{y}$ is the yaw rate, $\omega_{x}$ is the roll rate. $\delta_{x}, \delta_{y}$ and $\delta_{z}$ are the roll, yaw and pitch deflection angles, $a_{i}, b_{i}, c_{i}(i=1, \ldots, 8)$ are aerodynamic parameters, $f_{1}$ and $f_{2}$ are mismatched uncertainties vectors which are composed of external disturbance and internal parameter uncertainties, and satisfy $\left\|\boldsymbol{f}_{1}\right\| \leqslant d_{1}$ and $\left\|\boldsymbol{f}_{2}\right\| \leqslant d_{2}, d_{1}$ and $d_{2}$ are known constants.

Two ITSM surfaces are defined as

$$
\begin{aligned}
& \boldsymbol{s}_{1}=\boldsymbol{e}_{1}+\int_{0}^{t}\left[\boldsymbol{K}_{11} \boldsymbol{e}_{1}^{l}+\boldsymbol{K}_{12} \boldsymbol{e}_{1}+\varepsilon_{1} \operatorname{sgn}\left(\boldsymbol{e}_{1}\right)\right] \mathrm{d} t \\
& \boldsymbol{s}_{2}=\boldsymbol{e}_{2}+\int_{0}^{t}\left[\boldsymbol{K}_{21} \boldsymbol{e}_{2}^{l}+\boldsymbol{K}_{22} \boldsymbol{e}_{2}+\boldsymbol{\varepsilon}_{2} \operatorname{sgn}\left(\boldsymbol{e}_{2}\right)\right] \mathrm{d} t
\end{aligned}
$$

where $\boldsymbol{e}_{1}=\boldsymbol{x}_{1}-\boldsymbol{x}_{m}, \boldsymbol{x}_{m}$ is the desire attitude angle of vehicle, $\boldsymbol{e}_{2}=\boldsymbol{x}_{2}-\boldsymbol{x}_{2 c}, \boldsymbol{x}_{2 c}$ is virtual control and is defined as

$$
\begin{aligned}
& \boldsymbol{x}_{2 c}=-\boldsymbol{A}_{12}^{-1}\left[\boldsymbol{A}_{11} \boldsymbol{x}_{1}-\boldsymbol{K}_{11} \boldsymbol{e}_{1}^{l}+\boldsymbol{K}_{12} \boldsymbol{e}_{1}+\boldsymbol{\varepsilon}_{1} \operatorname{sgn}\left(\boldsymbol{e}_{1}\right)-\right. \\
& \left.\dot{\boldsymbol{x}}_{1 m}+\boldsymbol{H}_{11} \boldsymbol{s}_{1}^{l}+\boldsymbol{H}_{12} \boldsymbol{s}_{1}+\boldsymbol{\delta}_{1} \operatorname{sgn}\left(\boldsymbol{s}_{1}\right)\right] \\
& \boldsymbol{K}_{11}=\operatorname{diag}\left(k_{11 i}\right), \boldsymbol{K}_{12}=\operatorname{diag}\left(k_{12 i}\right), \boldsymbol{\varepsilon}_{1}=\operatorname{diag}\left(\varepsilon_{1 i}\right), \\
& k_{11 i}>0, k_{12 i}>0, \varepsilon_{1 i}>0, \boldsymbol{K}_{21}=\operatorname{diag}\left(k_{21 i}\right), \\
& \boldsymbol{K}_{22}=\operatorname{diag}\left(k_{22 i}\right), \boldsymbol{\varepsilon}_{2}=\operatorname{diag}\left(\varepsilon_{2 i}\right), k_{21 i}>0, k_{22 i}>0, \\
& \varepsilon_{2 i}>0, \boldsymbol{H}_{11}=\operatorname{diag}\left(h_{11 i}\right), \boldsymbol{H}_{12}=\operatorname{diag}\left(h_{12 i}\right), \boldsymbol{\delta}_{1}= \\
& \operatorname{diag}\left(\delta_{1 i}+d_{1}\right), h_{11 i}>0, h_{12 i}>0, \delta_{1 i}>0, i=1,2,3 .
\end{aligned}
$$

According to Theorem 2, the ITSM controller is chosen as

$$
\begin{gathered}
\boldsymbol{u}= \\
-\boldsymbol{B}^{-1}\left[\boldsymbol{A}_{21} \boldsymbol{x}_{1}+\boldsymbol{A}_{22} \boldsymbol{x}_{2}+\boldsymbol{K}_{21} \boldsymbol{e}_{2}^{l}+\boldsymbol{K}_{22} \boldsymbol{e}_{2}+\boldsymbol{\varepsilon}_{2} \operatorname{sat}\left(\boldsymbol{e}_{2}\right)-\right. \\
\left.\dot{\boldsymbol{x}}_{2 c}+\boldsymbol{H}_{21} \boldsymbol{s}_{2}^{l}+\boldsymbol{H}_{22} \boldsymbol{s}_{2}+\boldsymbol{\delta}_{2} \operatorname{sat}\left(\boldsymbol{s}_{2}\right)\right]
\end{gathered}
$$

where $\boldsymbol{H}_{21}=\operatorname{diag}\left(h_{21 i}\right), \boldsymbol{H}_{22}=\operatorname{diag}\left(h_{22 i}\right), \boldsymbol{\delta}_{2}=$ $\operatorname{diag}\left(\delta_{2 i}+d_{2}\right), h_{21 i}>0, h_{22 i}>0, \delta_{2 i}>0, i=1,2,3$.

Based on a hypersonic vehicle model, the mathematical simulation of the attitude control system is carried out, and the control effect is verified by tracking the step control instruction signal. Meanwhile, two control methods including TSM and MFTSM are also utilized in the simulation.

The initial flight speed of the aircraft is $6 \mathrm{Ma}$ and initial flight altitude is $30 \mathrm{~km}$. The desire square wave command of the angle of attack and roll are $5^{\circ}$, the desire sideslip angle is $0^{\circ}$. The parameters of the ITSM controller are follow as $k_{11 i}=4, k_{12 i}=2, k_{21 i}=0.8, k_{22 i}=0.8, \varepsilon_{1 i}=3$, $\varepsilon_{2 i}=0.1, h_{11 i}=2, h_{12 i}=2, h_{21 i}=1, h_{22 i}=1$, $\delta_{1 i}=0.5, \delta_{2 i}=0.1, l=5 / 3$. The amplitudes of the actuators are restricted as $-30^{\circ} \leqslant \delta_{x}, \delta_{y}, \delta_{z} \leqslant 30^{\circ}$.

As shown is Figs. 5-7, it is shown the response curve of vehicle angle of attack, side slip angle and roll angle under the three controllers. Angle of attack, sideslip angle and roll angle could track the desire values in finite time under the three controllers. However, the convergence time of the three attitude angles under the proposed ITSM controller is the fastest among the three controllers.

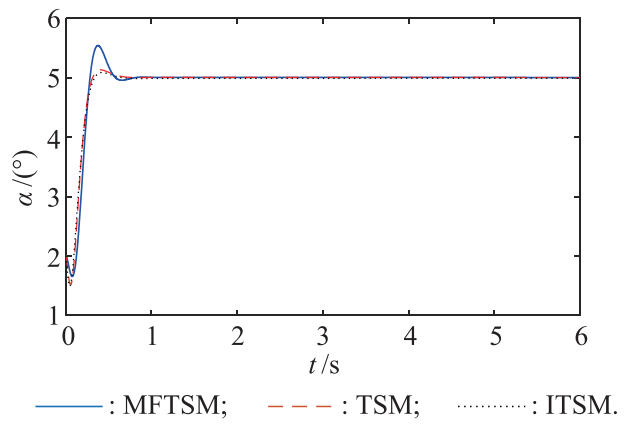

Fig. 5 Response curves of angle of attack 


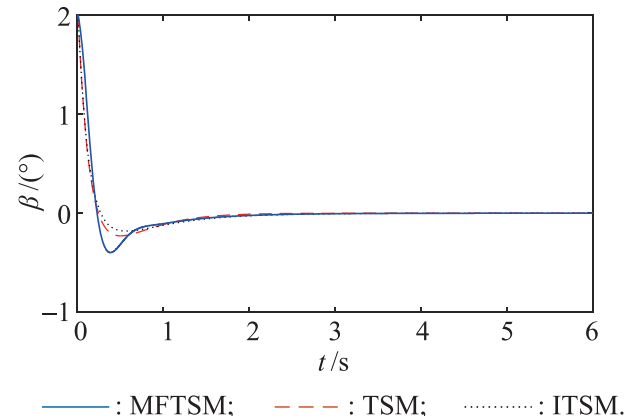

Fig. 6 Response curves of sideslip angle

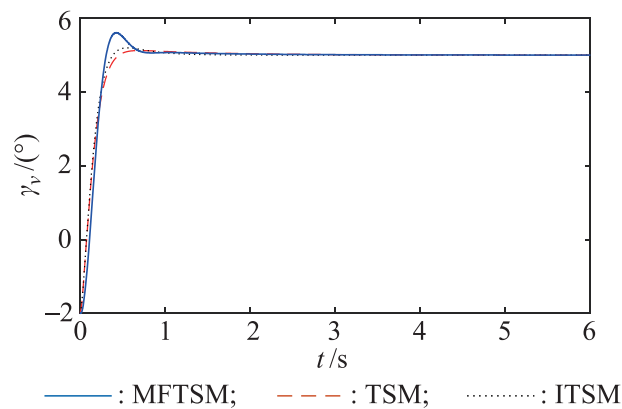

Fig. 7 Response curves of roll angle

The response curve of the roll, yaw and pitch deflection angles under the three controllers are shown in Figs. 8-10. Although, the maximum amplitude of the actuator in pitch and roll channel is reached, the attitude control system is still stable in finite time under the three controllers. Also, the control energy of the MFTSM controller is the minimum and that of the ITSM controller is the maximum.

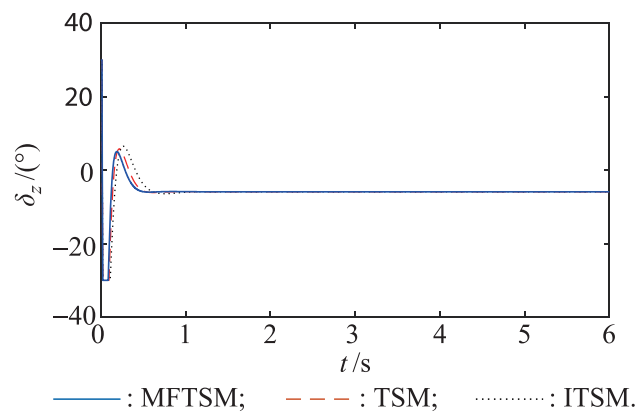

Fig. 8 Response curves of pitch deflection angle

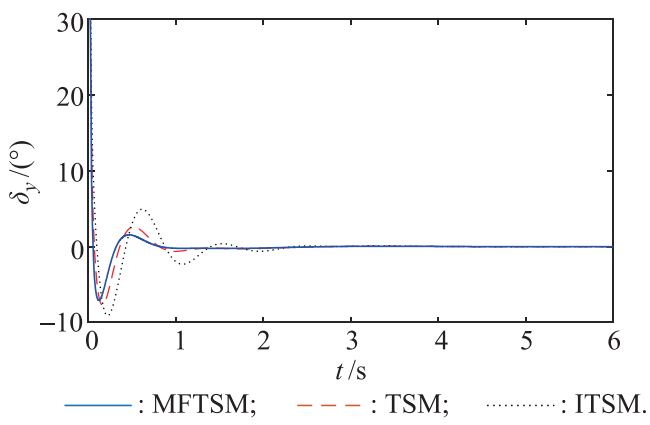

Fig. 9 Response curves of yaw deflection angle

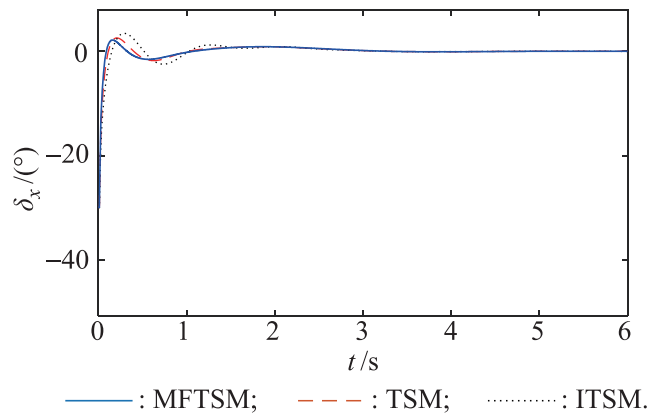

Fig. 10 Response curves of roll deflection angle

\section{Conclusions}

In this paper, a global nonsigular ITSM controller for $n$ order nonlinear systems with mismatched uncertainties has been proposed by using the backstepping technique. The time taken to reach the manifold from any initial system states and the time taken to reach the equilibrium point in the sliding mode have been proved to be finite. The new integral terminal sliding mode control is put forward, which can enable the elimination of the singularity problem associated with conventional terminal sliding mode control. Simulation results are presented to show the validity of the proposed ITSM control method.

\section{References}

[1] WANG Y, JIANG C, WU Q. Attitude tracking control for variable structure near space vehicles based on switched nonlinear systems. Chinese Journal of Aeronautics, 2013, 26(1): 186193.

[2] GUO C, LIANG X. Integrated guidance and control based on block backstepping sliding mode and dynamic control allocation. Proceeding of the Institution of Mechanical Engineering Part G: Journal of Aerospace Engineering, 2014, 229(9): $1559-1574$.

[3] SHAO X, WANG H. Active disturbance rejection based trajectory linearization control for hypersonic reentry vehicle with bounded uncertianties. ISA Transactions, 2015, 54: 27-38.

[4] VENKATARAMAN S T, GULATI S. Control of nonlinear systems using terminal sliding modes. ASME Journal of Dynamic Systems, Measurement and Control, 1993, 115(4): $554-560$.

[5] SONG Z K, LI H X, SUN K B. Finite-time control for nonlinear spacecraft attitude based on terminal sliding mode technique. ISA Transactions, 2014, 1(53): 117-124.

[6] AGHABABA M P, MOHAMMAD P. Design of hierarchical terminal sliding mode control scheme for fractional-order systems. IET Science Measurement \& Technology, 2015, 9(1): $122-133$

[7] KHARI S, RAHMANI Z, REZAIE B. Designing fuzzy logic controller based on combination of terminal sliding mode and state feedback controllers for stabilizing chaotic behaviour in rod-type plasma torch system. Transactions of the Institute of Measurement \& Control, 2016, 38(2): 150-164.

[8] ZHONG C X, GUO Y, YU Z. Finite-time attitude control for flexible spacecraft with unknown bounded disturbance. Transactions of the Institute of Measurement \& Control, 2016, 38(2): $240-249$. 
[9] FENG Y, YU X H, MAN Z. Non-singular terminal sliding mode control of rigid manipulators. Automatica, 2002, 38(12): $2159-2167$.

[10] YU S, YU X, SHIRINZADEH B, et al. Continuous finite-time control for manipulators with terminal sliding mode. Automatica, 2005, 41(11): 1957-1964.

[11] LIU G W, LI Z. Terminal sliding mode control for time-delay system with unmatched time-varying uncertainties. Systems Engineering and Electronics, 2009, 31(5): 1180-1183. (in Chinese)

[12] WANG L, CHAI T, ZHAI L. Neural-network-Based on terminal sliding mode control of robotic manipulators including actuator dynamics. IEEE Trans. on Industrial Electronics, 2009, 56(9): $3296-3304$.

[13] ZOU A, KUMAR K D, HOU Z, et al. Fintite-time attitude tracking control for spacecraft using terminal sliding mode and chebyshev neural network. IEEE Trans. on Systems, Man, and Cybernetics-Part B: Cybernetics, 2011, 41(4): 950-963.

[14] LU K, XIA Y. Adaptive attitude tracking control for rigid spacecraft with finite-time convergence. Automatica, 2013, 49(12): $3591-3599$.

[15] CHIU C. Derivative and integral terminal sliding mode control for a class of MIMO nonlinear systems. Automatica, 2012, 48(2): $316-326$.

[16] CASTAÑOS F, FRIDMAN L. Analysis and design of integral sliding manifolds for systems with unmatched perturbations. IEEE Trans. on Automatic Control, 2006, 51(5): 853-858.

[17] SCARRATT J C, ZINOBER A S I, MILLS R E, et al. Dynamical adaptive first and second-order sliding backstepping control of nonlinear nontriangular uncertain systems. ASME Journal of Dynamic Systems, Measurement and Control, 2000, 122(4): $746-752$.

[18] ZHANG J H, LIU X W, XIAN Y Q, et al. Disturbance observer based integral sliding mode control for systems with mismatched disturbances. IEEE Trans. on Industrial Electronics, 2016, 63(11): $7040-7048$.

[19] AVILA H H, LOUKIANOV A G, CAÑEDOC J M. Nested integral sliding modes of large sacale power systems. Proc. of the 46th IEEE Confernece on Decision Control, 2007: 1993 1998 .
[20] LEVANT A. Robust exact differentiation via sliding mode technique. Automatica, 1998, 34(3): 379-384.

[21] YU X, MAN Z. Fast terminal sliding mode control design for nonlinear dynamic systems. IEEE Trans. on Circuits and Systems I: Fundamental Theory and Applications, 2002, 49(2): $261-264$.

[22] TIE L, CAI K. A general form and improvement of fast terminal sliding mode. Proc. of the 8th World Congress on Intelligent Control and Automation, 2010: 2496-2501.

\section{Biographies}

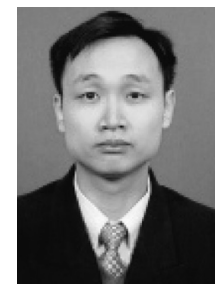

GUO Jianguo was born in 1975. He is a Ph.D. and a professor in School of Astronautics, Northwestern Polytechnical University. In the process of scientific research work, he studied on directions: precision guidance and control technology of aircraft; modern control theory and application.

E-mail: guojianguo@nwpu.edu.cn

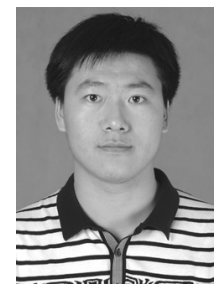

LIU Yuchao was born in 1990. He is a Ph.D. and gets his bachelor's degree and master's degree in School of Astronautics, Northwestern Polytechnical University. In the process of scientific research work, he studied on directions: precision guidance and control technology of aircraft, modern control theory and application.

E-mail: lyc-me@qq.com

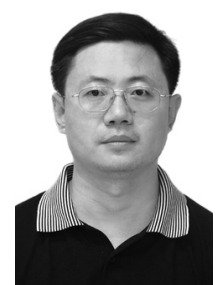

ZHOU Jun was born in 1966. He is a Ph.D. and a professor in School of Astronautics, Northwestern Polytechnical University. In the process of scientific research work, he gradually formed three main research directions: spacecraft control and simulation technology, modern control theory and application, precision guidance and control technology.

E-mail: zhoujun@nwpu.edu.cn 